\title{
Processing distributed inputs in coupled excitable lasers
}

\author{
Cristina M. González, ${ }^{1}$ Javier M. Buldú, ${ }^{2}$ M. C. Torrent, ${ }^{1}$ and Jordi García-Ojalvo ${ }^{1}$ \\ ${ }^{1}$ Departament de Física i Enginyeria Nuclear, Universitat Politècnica de Catalunya, Colom 11, E-08222 Terrassa, Spain \\ ${ }^{2}$ Nonlinear Dynamics and Chaos Group, Departamento de Física, Universidad Rey Juan Carlos, Tulipán s/n, 28933 Móstoles, \\ Madrid, Spain
}

(Received 30 March 2007; revised manuscript received 11 August 2007; published 19 November 2007)

\begin{abstract}
In many instances, networks of dynamical elements are subject to distributed input signals that enter the network through different nodes. In these cases, processing of the input signals may be mediated by coupling, in what constitutes an emerging property of the network. Here we study experimentally this effect in two mutually injected semiconductor lasers with optical feedback, operating in an excitable regime. The lasers are subject to different periodic input signals in their pump current, with distinct frequencies. Our results show that when the signals are harmonics of an absent fundamental, the laser array is able to process these signals and respond at the missing fundamental frequency. When the input frequencies are rigidly shifted from their harmonic values, the response frequency follows a simple law derived from a linear sum of the inputs, even though the array integrates the electrical inputs after having transduced them optically. The results are reproduced numerically with a dynamical model of the laser array.
\end{abstract}

DOI: 10.1103/PhysRevA.76.053824

PACS number(s): 42.65.Sf, 42.55.Px, 05.45.Xt, 89.75.Fb

\section{INTRODUCTION}

In both natural and technological settings, information processing is a task inherently undertaken by nonlinear systems $[1,2]$. Within that context, the response of nonlinear systems to simple harmonic signals has been widely studied [3]. Multifrequency signals have also been studied as a first approximation to complex inputs. In that case, phenomena such as vibrational resonance [4,5], subharmonic resonance [6], and frequency mixing [7] have been reported.

Many information-processing systems, such as the brain or telecommunication networks, are composed of multiple dynamical elements, which receive multiple signals of different frequencies at diverse input locations. It is therefore of interest to determine how coupling between the dynamical nodes of a processing network affects the integration of distributed signals. In this paper, we examine experimentally the relatively simple case of two mutually coupled dynamical elements, specifically two semiconductor lasers with optical feedback. Two external signals are applied to the pump currents of the lasers in the form of two periodic current modulations of dissimilar frequencies. As we show below, the laser array responds at a frequency not present in the input. This phenomenon, known as ghost resonance, is one of the most basic examples of information processing [8].

Ghost resonant behavior occurring in isolated dynamical elements has been reported experimentally in lasers $[9,10]$ and electronic circuits [11]. Recent experimental work has shown that the phenomenon also arises in a system of two coupled lasers [12]. In that case, however, the lasers were stable in the absence of coupling, and thus they cannot be considered separate dynamical elements. Here we study, on the other hand, the response of two coupled bona fide dynamical systems to a distributed signal. Recent studies in neuronal systems, both theoretical [13] and experimental [14], show that coupling is able to mediate the processing of distributed inputs in networks of neurons (which possess independent dynamics even in the absence of coupling). The present work constitutes experimental verification of this emerging property of excitable networks, using semiconductor lasers with optical feedback as highly controllable excitable systems $[15,16]$.

The paper is organized as follows. The next section provides a description of the experimental setup used. Section III discusses the dynamical behavior of the lasers with feedback in the absence of coupling and the effect of coupling on the collective dynamics of the system. Section IV shows that the coupled laser system is able to process a complex input signal composed of two harmonics of an absent fundamental, when these are distributed between the two lasers. Section V analyzes the response of the system when the input signals are no longer harmonics of the ghost frequency, showing that the system response is nontrivial. Section VI introduces a theoretical model that satisfactorily reproduces the phenomenon. This model is further used in Sec. VII to examine the influence of both coupling and delay, and resonant behavior is found with respect to these two parameters as well.

\section{EXPERIMENTAL SETUP}

Our experimental setup is represented in Fig. 1. Two semiconductor lasers LD1 and LD2 are mutually injected and subject to their own optical feedback from the external mirrors $M$. The coupling between the lasers can be controlled through a neutral density filter (NDF). The two AlGaInP index-guided and multi-quantum-well semiconductor lasers (Roithner RLT6505MG) operate at a nominal wavelength $\lambda$ $=650 \mathrm{~nm}$. Their temperature and pump current are controlled with an accuracy of $\pm 0.01{ }^{\circ} \mathrm{C}$ and $\pm 0.1 \mathrm{~mA}$, respectively. At temperatures $T_{\mathrm{LD} 1}=18.68{ }^{\circ} \mathrm{C}$ and $T_{\mathrm{LD} 2}=18.26^{\circ} \mathrm{C}$, the threshold currents of the lasers (in isolation) are, respectively, $I_{\mathrm{LD} 1}^{\mathrm{th}}=17.1 \mathrm{~mA}$ and $I_{\mathrm{LD} 2}^{\mathrm{th}}=16.6 \mathrm{~mA}$. The operating currents are set to $I_{\mathrm{LD} 1}=17.9 \mathrm{~mA}$ and $I_{\mathrm{LD} 2}=18.1 \mathrm{~mA}$. The relative pump currents, with respect to the threshold, are slightly different for both lasers, but this small asymmetry does not have an important influence in the results that follow. 


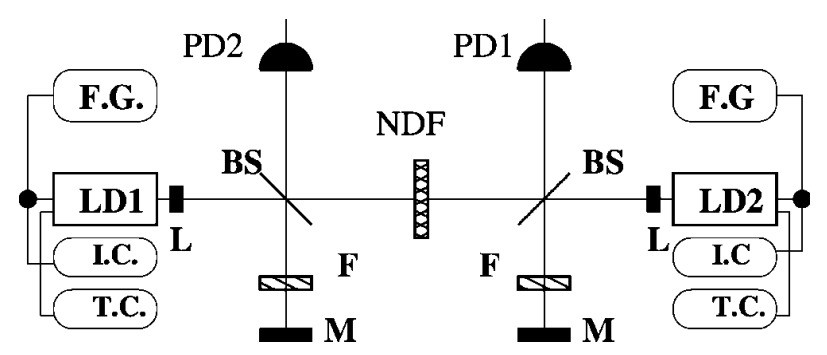

FIG. 1. Schematic setup. LD1 and LD2: laser diodes. TC and IC: temperature and current controllers. FG: function generators. $L$ : collimating lenses. BS: beam splitters. PD1 and PD2: photodetectors. $M$ : mirrors. $F$ : feedback filters. NDF: coupling filter.

Antireflection-coated objectives $(L)$ are used to collimate the light emitted by the two lasers. The external mirrors $M$ are placed $51 \mathrm{~cm}$ away from each laser, introducing a feedback delay time $\tau_{f}$ equal to the coupling time $\tau_{c}=3.4 \mathrm{~ns}$. Filters $F$ in front of each mirror diminish the quantity of reinjected light.

We position the external mirrors for optimum alignment, minimizing the laser's current threshold in the presence of optical feedback. This reduction is of 3.3\% for LD1 and $2.4 \%$ for LD2. To quantify the effect of the opposite laser as a source of additional feedback we estimate the threshold reduction when the opposite laser is turned off, obtaining a reduction of $1.2 \%$ for $\mathrm{LD} 1$ and $1.1 \%$ for $\mathrm{LD} 2$ with respect to the thresholds in isolation. When both lasers are turned on, the threshold reductions are $4.8 \%$ for LD1 and $4.2 \%$ for LD2. The laser outputs are monitored by two fast photodetectors PD1 and PD2 of $1 \mathrm{GHz}$ bandwidth (Thorlabs DET210). The received signal is sent to a $5 \mathrm{GS} / \mathrm{s}$ acquisition card (Gage $85 \mathrm{G}$ ), and to an spectrum analyzer (Anritsu MS2650B) via two amplifiers (2 GHz bandwidth, Femto high-speed amplifier).

\section{DYNAMICS IN THE ABSENCE OF COUPLING}

In isolation and for moderate feedback strengths, semiconductor lasers with optical feedback are known to have excitable properties, whereby small perturbations in the pump current produce large and brief dropouts in the emitted intensity $[15,16]$. An example of the dynamics of the two lasers in the absence of coupling is shown in Fig. 2(a). The plots show that the lasers undergo power dropouts at irregular times, driven by the underlying complex dynamics of the delayed system [17], which can be considered effectively as a noise source. Note that the $1 \mathrm{GHz}$ bandwidth of the detectors smoothes out the fast pulsing dynamics [18], resulting in a measurement of only the slower dropout envelope, which is the signal used in this study. When we start coupling the lasers by increasing the transmittivity of the neutral density filter, correlations between the power dropouts arise, until for the maximum coupling strength attainable in our experimental setup (when the NDF is removed) the lasers are fully synchronized [Fig. 2(b)]. In the results that follow, the lasers will be located in this regime. Synchronized dynamics in this experimental setup has been previously reported in Ref. [19].

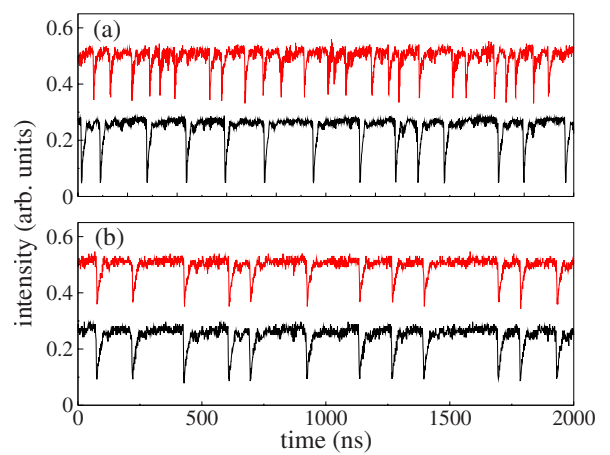

FIG. 2. (Color online) Output intensity of LD1 (top traces) and LD2 (bottom traces) without (a) and with (b) coupling. In (a), the output intensities are independent; in (b), the lasers are synchronized.

We have seen above that an isolated laser with feedback undergoes pulsed dynamics in the form of an irregular train of power dropouts. Applying a harmonic modulation to the laser's pump current allows us to control this irregular behavior, leading to a periodic train of dropouts at the frequency of the input signal [20]. This effect is most pronounced when the modulation frequencies are on the order of tens of $\mathrm{MHz}$, even though the typical characteristic frequencies of the laser are higher (the relaxation oscillation frequency without feedback is of the order of tens of $\mathrm{GHz}$, and the modes of the external cavity are of the order of hundreds of $\mathrm{MHz}$ ). This behavior can be explained from the fact that the periodic modulation impresses sidebands on each of the cavity mode and antimode frequencies [17], and a resonance occurs when the external frequency is equal to the modeantimode difference frequency. Exciting this resonance enhances the probability of inducing a dropout event. The optimal modulation frequency depends on the location in phase space where the trajectory resides the longest (see Refs. $[17,20]$ for details).

\section{SIGNAL PROCESSING MEDIATED BY COUPLING}

Coupling between two lasers was found to greatly enhance the responsivity of the system to the external harmonic driving [21]. We now apply two distributed inputs in the form of pump current modulations of different frequencies to each laser. The external modulations are introduced in the pump current of the lasers with two function generators (Agilent 33250A). First we choose two harmonics of a common fundamental $f_{0}$, defined by $f_{1}=k f_{0}$ and $f_{2}=(k+1) f_{0}$ with $k>1$. The behavior of the system for $k=2$ and $f_{0}=5 \mathrm{MHz}$ is shown in Fig. 3 for increasing amplitudes of the modulation, assumed equal for both signals. The figure shows the time trace of the intensity of LD1 on the left and the probability distribution of the interval between dropouts on the right (the results are basically identical for LD2, since both lasers are synchronized). The latter is computed from a collection of 1000 dropouts in each case. For a small modulation amplitude (top row in Fig. 3) the dropouts occur infrequently at different periods. As the amplitude grows (middle row), most 


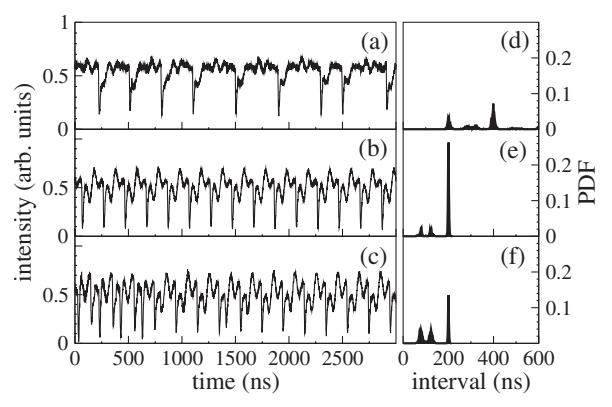

FIG. 3. Experimental output intensity of LD1 (left column) and the corresponding probability distribution functions of the time intervals between consecutive dropouts (right column) for increasing values of the modulation amplitude: (a),(d) $A_{1}=A_{2}=0.285 \mathrm{~mA}$; (b),(e) $A_{1}=A_{2}=0.643 \mathrm{~mA}$; (c),(f) $A_{1}=A_{2}=0.750 \mathrm{~mA}$. The input frequencies are $f_{1}=10 \mathrm{MHz}$ and $f_{2}=15 \mathrm{MHz}$, corresponding to interpulse periods $T_{1}=100 \mathrm{~ns}$ and $T_{2}=66.7 \mathrm{~ns}$. The ghost frecuency is $f_{0}=5 \mathrm{MHz}$, corresponding to a period $T_{0}=200 \mathrm{~ns}$.

interpulse intervals occur at a definite period corresponding to the fundamental frequency $f_{0}$, which is not present in either of the input signals. For larger amplitudes (bottom row), the input signals take over and dropouts begin to occur at the (larger) input frequencies, reducing the response of the system at the missing fundamental frequency. Therefore, a resonant behavior is observed with respect to the modulation strength: for an intermediate modulation amplitude, the system optimally processes the distributed inputs. We note that this resonance is nontrivially arising from the interplay between the direct electrical modulation of the pump current and the indirect optical driving coming from the other laser.

In the experimental conditions used, the lasers are detuned such that one of them consistently leads the dynamics, with a time lag equal to the coupling time [22]. The behavior of the system does not change if the input modulations are switched between the leader and laggard lasers. It is remarkable that the distributed signals are processed irrespective of

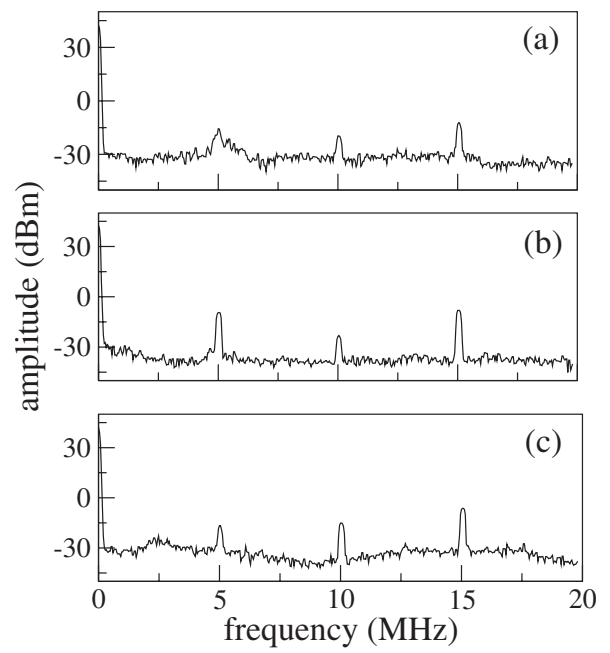

FIG. 4. Experimental rf spectrum of the output intensity of LD1 for increasing values of the modulation amplitude. Parameters are those of Fig. 3.

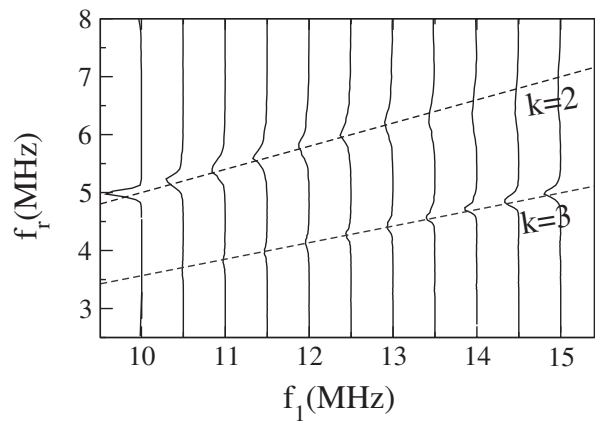

FIG. 5. Experimental distribution of response frequencies $f_{r}$ (vertically) versus the input frequency $f_{1}$ (and therefore $\Delta f$ ). The dashed lines correspond to the theoretical resonance frequencies given by Eq. (2).

this underlying asymmetry in the coupled dynamics.

The subharmonic resonance presented above can also be observed at the level of the rf spectrum of the lasers' outputs, as shown in Fig. 4. Peaks of the three frequencies involved, the two (higher) input frequencies $f_{1}=10 \mathrm{MHz}$ and $f_{2}$ $=15 \mathrm{MHz}$ and the fundamental frequency $f_{0}=5 \mathrm{MHz}$, are clearly observed in the spectrum. The height of the peaks at $f_{1}$ and $f_{2}$ increases monotonically with the modulation amplitude (from top to bottom), while the peak at $f_{0}$ is highest at an intermediate amplitude, which is a clear indicator of a resonance occurring at the missing fundamental frequency [8].

\section{INHARMONIC RESPONSE}

The results shown above do not correspond to a trivial resonance at the frequency difference $f_{2}-f_{1}$. To demonstrate this, we introduce a frequency shift $\Delta f$ in the input frequencies:

$$
f_{1}=k f_{0}+\Delta f, \quad f_{2}=(k+1) f_{0}+\Delta f .
$$

Such detuning renders the two input frequencies incommensurate and no longer harmonics of $f_{0}$. We kept the modulation amplitudes constant at $A_{1}=A_{2}=0.464 \mathrm{~mA}$ and varied the frequency of laser LD1 from $2 f_{0}=10 \mathrm{MHz}$ to $3 f_{0}=15 \mathrm{MHz}$ in steps of $0.5 \mathrm{MHz}$, while changing at the same time the frequency of laser LD2 from $3 f_{0}=15 \mathrm{MHz}$ to $4 f_{0}=20 \mathrm{MHz}$, keeping $f_{2}=f_{1}+f_{0}$. Figure 5 shows that in this case the response frequency, defined as the inverse of the interval between two consecutive dropouts, increases linearly with the detuning, even though the difference between the input frequencies remains equal to $f_{0}$. The maxima of the response frequency distribution obey the following simple relation:

$$
f_{r}=f_{0}+\frac{\Delta f}{k+1 / 2},
$$

which was obtained in Ref. [6] from a straightforward analysis of the maxima of the linear superposition of two sinusoidal functions with frequencies given by Eq. (1).

\section{NUMERICAL SIMULATIONS}

We modeled the phenomena described above with a generalized version of the Lang-Kobayashi delay differential 


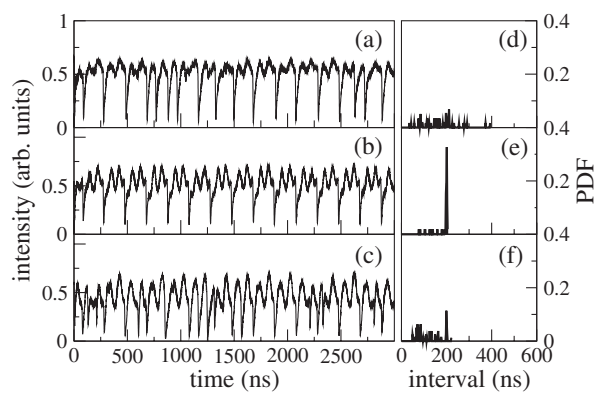

FIG. 6. Numerical output intensity of LD1 (left column) and the corresponding probability distribution functions (right column) for increasing values of the modulation amplitude: (a),(d) $A_{1}=A_{2}=0.004$; (b),(e) $A_{1}=A_{2}=0.006$; and (c),(f) $A_{1}=A_{2}=0.008$. The parameters are $\alpha=3.0, \quad \beta=0.5 \times 10^{-9} \mathrm{ps}^{-1}, \quad \kappa_{c}=20 \mathrm{~ns}^{-1}$, $\kappa_{f}=10 \mathrm{~ns}^{-1}, \quad \gamma_{1}=0.50 \mathrm{ps}^{-1}, \quad \gamma_{2}=0.48 \mathrm{ps}^{-1}, \quad \gamma_{e 1}=6.89 \times 10^{-4} \mathrm{ps}^{-1}$, $\gamma_{e 2}=6.72 \times 10^{-4} \mathrm{ps}^{-1}, \quad g_{1}=1.20 \times 10^{-9} \mathrm{ps}^{-1}, \quad g_{2}=1.18 \times 10^{-9} \mathrm{ps}^{-1}$, $N_{1}^{0}=1.25 \times 10^{8}$, and $\tau_{c}=\tau_{f}=3.43 \mathrm{~ns}$.

model, describing the behavior of two bidirectionally coupled lasers with feedback [23]:

$$
\begin{aligned}
\frac{d E_{1,2}}{d t}= & \frac{(1+i \alpha)}{2}\left[G_{1,2}-\gamma_{1,2}\right] E_{1,2}+\kappa_{c} e^{i \Omega \tau_{c}} E_{2,1}\left(t-\tau_{c}\right) \\
& +\kappa_{f} e^{-i \omega_{1,2} \tau_{f}} E_{1,2}\left(t-\tau_{f}\right)+\sqrt{2 \beta N_{1,2}} \xi_{1,2}\left(\tau_{f}\right), \\
& \frac{d N_{1,2}}{d t}=\frac{I_{1,2}}{e}-\gamma_{e 1,2} N_{1,2}-G_{1,2} P_{1,2}(t),
\end{aligned}
$$

where $E_{1,2}$ represent the optical fields of LD1 and LD2 and $N_{1,2}$ their corresponding carrier numbers. $\omega_{1,2}$ are the freerunning optical frequencies of the lasers, which for simplicity are considered to be the same, so that $\Omega=\omega_{2}-\omega_{1}=0$. The optical intensity (or number of photons inside the cavity) is given by $P_{1,2}(t)=\left|E_{1,2}(t)\right|^{2}$. The first term on the right-hand side of Eq. (3) accounts for stimulated emission. $G_{1,2}(t)$ $=g_{1,2}\left(N_{1,2}-N_{1,2}^{0}\right)$ is the nonlinear gain, with $N_{1,2}^{0}$ denoting the carrier number at transparency and $g_{1,2}$ the differential gain (gain saturation is neglected because the lasers operate close to threshold). The linewidth enhancement factor $\alpha$ is assumed to be the same for the two lasers. The inverse photon lifetime is denoted by $\gamma_{1,2}$. The second term in Eq. (3) accounts for the bidirectional coupling between the lasers, with $\kappa_{c}$ representing the coupling strength and $\tau_{c}$ the coupling time. The third term represents the feedback, which is described by two parameters: the feedback strength $\kappa_{f}$ and the external round-trip time $\tau_{f}$, which are also assumed equal for the two lasers. The last term accounts for the spontaneous emission noise, represented by a Gaussian white noise source of zero mean and correlation, $\left\langle\xi(t) \xi\left(t^{\prime}\right)\right\rangle=2 \delta\left(t-t^{\prime}\right)$, with spontaneous emission rate $\beta$. The first term in Eq. (4) corresponds to the injection current, the second term accounts for the spontaneous recombination, with the carrier inverse lifetime $\gamma_{e 1,2}$, and the third term corresponds to stimulated recombination.

We chose parameter values that reproduce the experimental conditions (given in the caption of Fig. 6). In

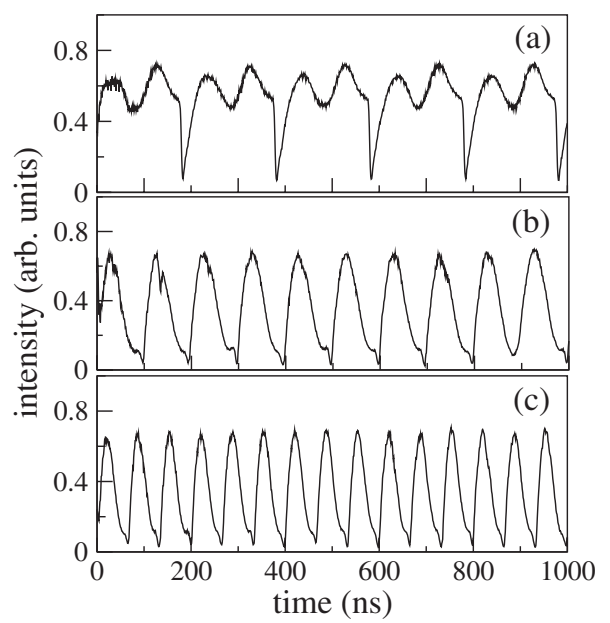

FIG. 7. Response of the coupled lasers to the complex harmonic signal (a) compared with the individual responses in the absence of coupling (b),(c). Parameters are those of Figs. 6(b) and 6(e), with $\kappa_{c}=0$ in panels (b) and (c).

particular, the pump currents take the form $I_{1,2}=I_{1,2}^{\mathrm{dc}}\left[1+A_{1,2} \sin \left(2 \pi f_{1,2} t\right)\right]$, where $I_{1,2}^{\mathrm{dc}}$ are the dc pump currents, $A_{1,2}$ are the modulation amplitudes, and $f_{1,2}$ are their corresponding frequencies, chosen again following Eq. (1). The dc levels are taken to be $I_{1}^{\mathrm{dc}}=1.058 \times I_{\mathrm{LD} 1}^{\mathrm{th}}, I_{2}^{\mathrm{dc}}$ $=1.076 \times I_{\mathrm{LD} 2}^{\mathrm{th}}$.

Figure 6 shows the results obtained numerically in the harmonic $(\Delta f=0)$ case, with $f_{1}=10 \mathrm{MHz}$ and $f_{2}=15 \mathrm{MHz}$.

For low amplitudes the dropouts are not entrained, while for intermediate amplitudes the response at the missing fundamental frequency increases. For even higher modulation amplitudes, the peak at $f_{0}$ diminishes again.

To emphasize the fact that the response at the missing fundamental frequency is mediated by coupling, we compare in Fig. 7 the response of the system with and without coupling. With coupling both lasers are synchronized [panel (a), only one of the lasers is shown] and the system responds at the fundamental period (200 ns). On the other hand, when the lasers are isolated from each other (middle and bottom rows) only the corresponding periods of the individual modulations (66.6 ns and $100 \mathrm{~ns}$ ) are detected. In that case, the external driving leads to a strong periodic component in the laser output, as shown in panels (b) and (c) of Fig. 7, which is strongly reduced in the coupled case [Fig. 7(a)].

\section{INFLUENCE OF COUPLING AND FEEDBACK STRENGTHS}

The fact that the lasers have excitable dynamics even in the absence of coupling (see Sec. III) allows us to study the effect of coupling on the signal processing efficiency. To that end, we fix the amplitude of the input modulation to its optimal level, as obtained above, and maintain a constant level of feedback as well. The response of the system for increasing levels of coupling is shown numerically in Fig. 8. In the absence of coupling the lasers exhibit independent dynamics, each responding to its input frequency [Fig. 8(a)]. As the 


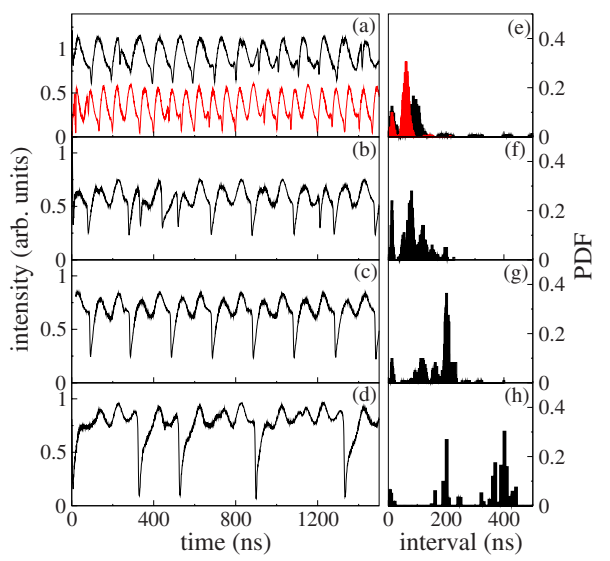

FIG. 8. (Color online) Output intensities and probability distribution function of the time interval between dropouts for increasing coupling strengths: (a),(e) $\kappa_{c}=0 \mathrm{~ns}^{-1}$; (b),(f) $\kappa_{c}=10 \mathrm{~ns}^{-1}$; (c),(g) $\kappa_{c}=20 \mathrm{~ns}^{-1}$; and (d),(h) $\kappa_{c}=40 \mathrm{~ns}^{-1}$. In panels (a) and (e) the results corresponding to the lasers are shown; in panel (a), the time trace of laser 1 has been shifted vertically for clarity. In panels (b)-(d) and (f)-(h) only the output of only one laser is shown, since the lasers are synchronized. Other parameters are those of Fig. 6.

coupling is increased a response at the ghost frequency (corresponding again to a 200-ns interval between dropouts) appears, becoming optimal for an intermediate coupling level [Fig. 8(c)].

Two features stand out from the results shown in Fig. 8 . First, synchronization of the lasers is not sufficient to produce a response at the ghost frequency. This is shown, for instance, in Figs. 8(b) and 8(f), where coupling is strong enough to synchronize the lasers' outputs (and thus only one time trace is shown in the figure), but is not strong enough to produce a ghost response. Second, for very strong coupling the ghost response disappears again, because the two lasers behave essentially as a single unit subject to two input modulations [9] and the system parameters have been chosen in such a way that the ghost response is not present for a single laser. This leads to a nonmonotonic behavior of the system's response with respect to coupling strength and a need to optimize that parameter in order to observe the phenomenon.

We also analyzed numerically the influence of the feedback strength on the system response for a coupling strength fixed to the optimal level determined in the previous paragraphs. As shown in Fig. 9, the feedback strength also needs to be optimized in order to obtain a response of the system at the ghost frequency. For low feedback levels [Figs. 9(a) and 9(d)], both lasers respond preferentially to the two input frequencies. It is worth noting that the lasers have already synchronized their dynamics at this point, but nevertheless they cannot process the input signals adequately. As the feedback level increases (identically for the two lasers), a joint response arises at the ghost frequency (corresponding again to a time interval between dropouts of $200 \mathrm{~ns}$ ). The response is lost once more at high feedback levels, revealing another resonance, this time with respect to the feedback level. This nonmonotonic behavior is due to the fact that the feedback

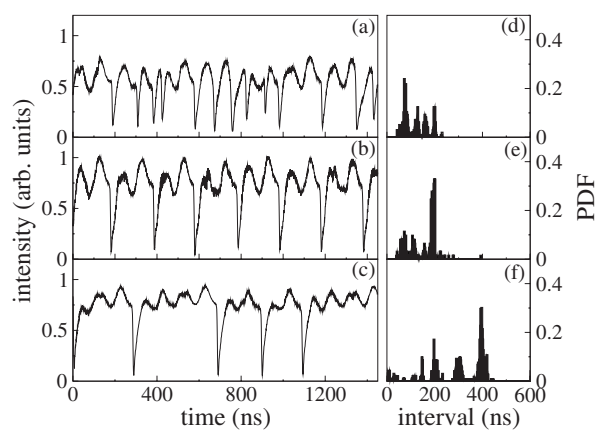

FIG. 9. Output intensities and probability distribution function of time interval between dropouts for increasing feedback levels: (a),(d) $\kappa_{f}=5 \mathrm{~ns}^{-1}$; (b),(e) $\kappa_{f}=10 \mathrm{~ns}^{-1}$; (c),(f) $\kappa_{f}=30 \mathrm{~ns}^{-1}$. The lasers are synchronized, so only one time trace is shown. The coupling strength is $\kappa_{c}=20 \mathrm{~ns}^{-1}$. Other parameters are those of Fig. 6 .

strength affects the response of each laser to the external modulation [20] in such a way that tuning the feedback level varies the sensibility of each laser to a given input frequency range. A ghost response can only arise when the feedback levels are tuned such that the two lasers respond preferentially to the ghost frequency.

\section{CONCLUSION}

In conclusion, we have shown experimentally that coupling between two excitable systems, specifically two semiconductor lasers with optical feedback, is able to mediate the processing of distributed inputs, applied in the form of pump current modulations of different frequencies. For large enough coupling strengths, the laser outputs have the form of synchronized trains of power dropouts which become entrained to a frequency that is not present in the input signals. When these signals are harmonics of a missing fundamental, the response occurs at precisely the fundamental frequency. On the other hand, in the case where the two input frequencies are equally shifted from these harmonics, the system responds following a linear law, which arises from analyzing the linear summation of the two input modulations [6]. The superposition law holds even though in our case the two signals acting upon a given laser are clearly different, one of them being electrical (through direct pump current modulation) and the other optical (through injection from the other laser). This type of response to complex input signals has been reported in the human brain with psycophysical experiments [24] and magnetoencephalographic recordings [25], which highlights the importance of understanding the integration of distributed inputs by networks of information processing elements.

\section{ACKNOWLEDGMENTS}

This research was supported by the Ministerio de Educacion y Ciencia (Spain, Project No. FIS2006-11452), by the Generalitat de Catalunya, and by the European Commission (GABA project, FP6-NEST Contract No. 043309). 
[1] D. Cotter, R. J. Manning, K. J. Blow, A. D. Ellis, A. E. Kelly, D. Nesset, I. D. Phillips, A. J. Poustie, and D. C. Rogers, Science 286, 1523 (1999).

[2] M. J. Chacron, J. Neurophysiol. 95, 2933 (2006).

[3] L. Glass and J. Sun, Phys. Rev. E 50, 5077 (1994), and references therein.

[4] P. Landa and P. McClintock, J. Phys. A 33, L433 (2000).

[5] E. Ullner, A. Zaikin, J. Garcia-Ojalvo, R. Bascones, and J. Kurths, Phys. Lett. A 312, 348 (2003).

[6] D. R. Chialvo, O. Calvo, D. L. Gonzalez, O. Piro, and G. V. Savino, Phys. Rev. E 65, 050902(R) (2002).

[7] J. H. E. Cartwright, D. L. González, and O. Piro, Phys. Rev. Lett. 82, 5389 (1999).

[8] D. R. Chialvo, Chaos 13, 1226 (2003).

[9] J. M. Buldú, D. R. Chialvo, C. R. Mirasso, M. C. Torrent, and J. García-Ojalvo, Europhys. Lett. 64, 178 (2003).

[10] G. Van der Sande, G. Verschaffelt, J. Danckaert, and C. R. Mirasso, Phys. Rev. E 72, 016113 (2005).

[11] O. Calvo and D. R. Chialvo, Int. J. Bifurcation Chaos Appl. Sci. Eng. 16, 731 (2006).

[12] J. M. Buldú, C. M. González, J. Trull, M. C. Torrent, and J. García-Ojalvo, Chaos 15, 013103 (2005).

[13] P. Balenzuela and J. García-Ojalvo, Chaos 15, 023903 (2005).
[14] E. Manjarrez et al., BioSystems 90, 379 (2007).

[15] M. Giudici, C. Green, G. Giacomelli, U. Nespolo, and J. R. Tredicce, Phys. Rev. E 55, 6414 (1997).

[16] J. Mulet and C. R. Mirasso, Phys. Rev. E 59, 5400 (1999).

[17] T. Sano, Phys. Rev. A 50, 2719 (1994); G. H. M. van Tartwijk, A. M. Levine, and D. Lenstra, IEEE J. Sel. Top. Quantum Electron. 1, 466 (1995).

[18] I. Fischer, G. H. M. van Tartwijk, A. M. Levine, W. Elsässer, E. Göbel, and D. Lenstra, Phys. Rev. Lett. 76, 220 (1996).

[19] E. Klein, N. Gross, M. Rosenbluh, W. Kinzel, L. Khaykovich, and I. Kanter, Phys. Rev. E 73, 066214 (2006).

[20] D. W. Sukow and D. J. Gauthier, IEEE J. Quantum Electron. 36, 175 (2000).

[21] J. M. Buldú, R. Vicente, T. Pérez, C. R. Mirasso, M. C. Torrent, and J. García-Ojalvo, Appl. Phys. Lett. 81, 5105 (2002).

[22] T. Heil, I. Fischer, W. Elsäßer, J. Mulet, and C. R. Mirasso, Phys. Rev. Lett. 86, 795 (2001).

[23] J. Mulet, C. Masoller, and C. R. Mirasso, Phys. Rev. A 65, 063815 (2002).

[24] J. F. Schouten, R. J. Ritsma, and B. L. Cardozo, J. Acoust. Soc. Am. 34, 1418-1424 (1962).

[25] C. Pantev, T. Elbert, B. Ross, C. Eulitz, and E. Terhardt, Hear. Res. 100, 164 (1996). 